\section{Early age-related maculopathy in eyes after cataract surgery}

${ }^{1}$ Department of

Ophthalmology (Centre for Vision Research, Westmead Millennium Institute), Westmead Hospital, University of Sydney, Westmead, NSW, Australia

\section{${ }^{2}$ Department of}

Ophthalmology, Westmead Hospital, Westmead, NSW, Australia

Correspondence: JJ Wang, Department of

Ophthalmology (Centre for Vision Research, Westmead Millenium Institute), Westmead Hospital, University of Sydney, Hawkesbury Road, Westmead, NSW 2145, Australia

Tel: +61 29845 5006; Fax: + 61298458345

E-mail: jiejin_wang@

wmi.usyd.edu.au

Received: 3 November 2005 Accepted: 7 December 2005

Published online: 27 January 2006

Commercial relationship: None

\begin{abstract}
Purpose To assess age-related maculopathy (ARM) in eyes of patients who had undergone cataract surgery for at least a year.

Methods Consecutive patients aged $60+$ years who had undergone cataract surgery at Westmead Hospital, Sydney, Australia, during 2001-2003 were examined in 2004. Interview using standardized questionnaires and stereo retinal photography was performed. Retinal photographs were graded using the Wisconsin ARM grading system. The proportions with ARM were compared between surgical and nonsurgical eyes, and between this surgical cohort and the Blue Mountains Eye Study (BMES) population.
\end{abstract}

Results Of the 622 eligible patients, 454 (73\%) were re-examined, with a mean followperiod of 2.8 years. Surgical eyes had a higher proportion of early ARM compared to nonsurgical eyes (15.2 vs $10.3 \%, P=0.07)$ and to the early ARM prevalence found in BMES participants of similar age (14.5 vs 6.9\%, $P<0.01$ ), which persisted after age standardization to the BMES population (9.7 vs $6.9 \%, P<0.05$ ).

Conclusions We found an increased prevalence of early ARM in surgical eyes of patients 1-3 years after cataract surgery. Whether this increased early ARM prevalence leads to an increased prevalence of late ARM in the long-term warrants further investigation.

Eye (2007) 21, 512-517. doi:10.1038/sj.eye.6702254; published online 27 January 2006

Keywords: cataract surgery; age-related maculopathy; prevalence; Blue Mountains Eye Study

\section{Introduction}

Cataract and late age-related maculopathy (ARM) are the two leading causes of visual
TQ Pham', S Cugati', E Rochtchina', P Mitchell', A Maloof ${ }^{2}$ and JJ Wang ${ }^{1}$

impairment in the elderly worldwide. ${ }^{1-5}$ While the cataract-induced visual impairment is mostly successfully reversed by cataract surgery, there are limited treatment options for both early and late stages of ARM.

On-going clinical observations have raised concerns that the risk of late ARM could be increased in eyes after cataract surgery. Although earlier case series ${ }^{6-9}$ and a postmortem study ${ }^{10}$ suggested a possible association between aphakia or pseudo-phakia and late ARM, the evidence from these studies is relatively weak. Findings from large population-based studies ${ }^{11,12}$ appeared to support an association between prior cataract surgery and an increase in late ARM prevalence. The strongest evidence to date, came from a pooled data analysis of two large populationbased studies ${ }^{13}$ that showed a significantly increased 5-year incidence of late ARM (odds ratio (OR) 5.7, 95\% confidence interval (CI) 2.4-13.6) in nonphakic (aphakic or pseudo-phakic) eyes compared to phakic eyes, after accounting for age, smoking, and the presence and severity of early ARM lesions at baseline. ${ }^{13}$ The Beaver Dam Eye Study (BDES) has reported a significant association between cataract surgery performed prior to baseline and the incidence of late ARM after both $5^{-14}$ and 10 -year ${ }^{15}$ intervals. The potential for an increased late ARM risk after cataract surgery remains topical and controversial, as indicated by two recent editorials published in Archives of Ophthalmology. ${ }^{16,17}$

While the increased risk of late ARM in nonphakic (surgical) eyes was observed longterm (at least 5 years) after cataract surgery, the BDES also reported that persons who had cataract surgery between baseline and the 5-year follow-up had higher age-adjusted incidence of early ARM detected at the 5-year examination. ${ }^{14}$ This suggested that early ARM may be more prevalent in the short to medium term following surgery and prior to subsequent development of late ARM. In order to confirm 
this hypothesis, we examined patients who had undergone cataract surgery in an Australian public hospital and assessed the prevalence of early ARM in surgical eyes of patients after a short- to medium-term period (1-3 years) of postoperative follow-up, and compared this to the prevalence in the fellow nonsurgical eye and to that in a large population-based study.

\section{Methods}

Westmead Hospital is part of the Sydney West Area Health Service and provides public cataract surgical services to residents living in mid-western areas of Sydney, Australia. In this hospital-based study sample, consecutive patients aged $60+$ years who had undergone cataract surgery at Westmead Hospital from July 2001 to June 2003 were included. Study procedures were approved by the hospital Human Research Ethics Committee and written, informed consent was obtained from all participants.

Preoperative information was obtained from a retrospective review of patient medical records. Baseline data collected included patient's demographic details (age, sex, and ethnicity), past medical history, smoking status, and preoperative macular status. During the period between July 2001 and June 2003, 941 routine cataract surgical procedures were performed. Of these, 784 procedures were performed on 709 patients aged 60 years or older at the time of surgery. Medical records were available on 718 procedures performed on 671 patients, who were considered potential participants for the follow-up examinations; 454 patients (73\% of survivors) were able to be re-examined, after excluding 49 patients who had died prior to the time of follow-up examinations. These deaths were confirmed either from medical records or by telephone contact with the next of kin.

All participants were examined between March and December 2004. The study examination included an examiner-administered interview, visual acuity examination, and stereo retinal photography after pupil dilation. Stereoscopic $35^{\circ}$ retinal photographs were taken from both eyes of participants, using a Topcon TRC 50 IA retinal camera (Topcon Corporation, Tokyo, Japan) and Kodak Ektachrome 64 35-mm slide film (Kodak, Melbourne, Australia). Photographs were centred on the macula and optic disc (Diabetic Retinopathy Study ${ }^{18}$ fields 2 and 1).

Masked grading for ARM lesions followed the Blue Mountain Eye Study (BMES), ${ }^{19}$ modification of the Wisconsin Age-Related Maculopathy Grading System. ${ }^{20}$ Late ARM was defined to include the two end-stage lesions, geographic atrophy and neovascular maculopathy. Early ARM was defined as the absence of late ARM and either (1) large $(>125 \mu \mathrm{m})$ indistinct soft drusen or reticular drusen, or (2) both large soft distinct drusen and retinal pigment epithelial (RPE) abnormalities (hyper- or hypopigmentation), within the superimposed grading grid. ${ }^{19}$ The component lesions for early ARM (soft drusen or retinal pigmentary abnormalities) were also assessed separately.

Statistical analyses were performed using the Statistical Analysis System (version 8.2, SAS Institute, Cary, NC, USA). For patients who had had only one eye operated at the time of the examination, the nonoperated eye was used as paired control for eye-specific prevalence comparisons. For person-specific prevalence comparisons, we used the worse eye to define ARM in persons who had had both eyes operated at the time of the follow-up examination. The prevalence of ARM from a general older $(60+$ years $)$ Australian population subgroup, a subgroup of this age from the BMES baseline survey (1992-4), ${ }^{21}$ was used as a reference, before and after direct age standardization. Proportions and ageadjusted $P$-value are presented.

\section{Results}

Table 1 shows a comparison of the baseline characteristics of surviving patients examined in the follow-up study $(n=454)$ with patients who were not examined ( $n=168$ ) or had died before the follow-up examination $(n=49)$. The mean age of participants was $76(\mathrm{SD} \pm 7.0)$ years. Patients who had moved out of the area or who refused to participate were not significantly different from the study group in terms of age or history of major illnesses. Those who had died before the examination were more likely to be older, to have had ischaemic heart disease, and less likely to have been current or past smokers. The ethnicities of those examined are also shown with the majority being Caucasian $(74 \%)$ plus other major ethnic groups of Middle Eastern, Asian, and Indian origin.

The mean postcataract surgery follow-up period of the 454 patients was 2.8 years, with a minimum of 0.9 years and maximum of 3.4 years. There were 179 patients (92 right and 87 left eyes) who had been operated on only one eye at the time of the follow-up examinations; 150 of the 179 had gradable photographs of both eyes for assessment of late ARM lesions and 145 for assessment of early ARM. Table 2 shows a comparison of the proportions with ARM lesions between surgical and nonsurgical eyes in this group by age group. There was an increased prevalence of both early and late ARM with increasing age in both the surgical and nonsurgical eyes. The proportion with late ARM was nonsignificantly higher in nonsurgical than surgical eyes (2.0 vs 1.3\%, $P=0.31$ ). The proportions with early ARM or early ARM component lesions (RPE abnormalities and soft indistinct 
Table 1 Comparison of baseline characteristics of participants and nonparticipants at the follow-up examination

\begin{tabular}{|c|c|c|c|c|c|}
\hline \multirow[b]{3}{*}{ Baseline characteristics } & \multicolumn{5}{|c|}{$\%$ of Participants and nonparticipants } \\
\hline & \multirow[b]{2}{*}{$\begin{array}{l}\text { Participated follow-up } \\
\quad \text { exams }(\mathrm{n}=454)\end{array}$} & \multicolumn{4}{|c|}{ Nonparticipants } \\
\hline & & $\begin{array}{l}\text { Refused } \\
(\mathrm{n}=168)\end{array}$ & $\begin{array}{c}\text { Age-adjusted } \\
\text { P-value }\end{array}$ & $\begin{array}{c}\text { Died } \\
(\mathrm{n}=49)\end{array}$ & $\begin{array}{c}\text { Age-adjusted } \\
\text { P-value }\end{array}$ \\
\hline \multicolumn{6}{|l|}{ Age (years) } \\
\hline $60-69$ & 29.7 & 24.4 & 0.15 & 14.3 & $<0.001$ \\
\hline $70-79$ & 50.2 & 48.8 & & 36.7 & \\
\hline $80+$ & 20.1 & 26.8 & & 49.0 & \\
\hline Female & 60.6 & 67.3 & 0.13 & 65.3 & 0.52 \\
\hline History of IHD & 29.7 & 28.0 & 0.67 & 42.9 & 0.06 \\
\hline History of stroke & 6.2 & 10.1 & 0.09 & 8.2 & 0.59 \\
\hline History of diabetes & 27.8 & 31.6 & 0.35 & 34.7 & 0.31 \\
\hline History of hypertension & 57.7 & 61.3 & 0.42 & 61.2 & 0.64 \\
\hline Past smoking & 40.5 & 25.0 & 0.001 & 16.3 & 0.001 \\
\hline Current smoking & 13.2 & 15.5 & 0.72 & 6.1 & 0.03 \\
\hline Presence of late ARM & 0.9 & 2.4 & 0.14 & 0 & \\
\hline \multicolumn{6}{|l|}{ Ethnicity } \\
\hline Caucasian & 74.0 & & & & \\
\hline Middle Eastern & 9.3 & & & & \\
\hline Asian & 6.8 & & & & \\
\hline Indian & 3.5 & & & & \\
\hline Other & 6.3 & & & & \\
\hline
\end{tabular}

$\mathrm{IHD}=$ ischaemic heart disease; $\mathrm{ARM}=$ age-related maculopathy.

Table 2 Comparison of proportions with age-related maculopathy (ARM) in surgical and non-surgical eyes of sample by age group, among participants with only one eye operated

\begin{tabular}{|c|c|c|c|c|c|c|c|c|c|}
\hline \multirow[t]{3}{*}{ Characteristics, eye } & \multicolumn{8}{|c|}{ Age group (years) } & \multirow[b]{3}{*}{ P-value } \\
\hline & \multicolumn{2}{|c|}{$60-69$} & \multicolumn{2}{|c|}{$70-79$} & \multicolumn{2}{|c|}{$80+$} & \multicolumn{2}{|c|}{ Total } & \\
\hline & $\mathrm{n}$ & $\%$ & $\mathrm{n}$ & $\%$ & $\mathrm{n}$ & $\%$ & $\mathrm{n}$ & $\%$ & \\
\hline \multicolumn{10}{|l|}{ Late ARM } \\
\hline Surgical eye $(n=150)$ & 0 & 0 & 0 & 0 & 2 & 4.7 & 2 & 1.3 & 0.31 \\
\hline Nonsurgical eye $(n=150)$ & 0 & 0 & 1 & 1.3 & 2 & 4.7 & 3 & 2.0 & \\
\hline \multicolumn{10}{|l|}{ Early ARM } \\
\hline Surgical eye $(n=145)$ & 2 & 6.7 & 5 & 6.6 & 15 & 38.5 & 22 & 15.2 & 0.07 \\
\hline Nonsurgical eye $(n=145)$ & 1 & 3.3 & 3 & 4.0 & 11 & 28.2 & 15 & 10.3 & \\
\hline \multicolumn{10}{|l|}{ RPE abnormalities } \\
\hline Surgical eye $(n=145)$ & 6 & 20.0 & 13 & 17.1 & 15 & 38.5 & 34 & 23.5 & $<0.01$ \\
\hline Nonsurgical eye $(n=145)$ & 4 & 13.3 & 3 & 4.0 & 12 & 30.8 & 19 & 13.1 & \\
\hline \multicolumn{10}{|c|}{ Indistinct soft drusen/reticular drusen } \\
\hline Surgical eye $(n=145)$ & 1 & 3.3 & 4 & 5.3 & 15 & 38.5 & 20 & 13.8 & 0.08 \\
\hline Nonsurgical eye $(n=145)$ & 1 & 3.3 & 3 & 4.0 & 10 & 25.6 & 14 & 9.7 & \\
\hline
\end{tabular}

$\mathrm{RPE}=$ retinal pigment epithelial.

or reticular drusen), however, were higher in the surgical than nonsurgical eyes across all age groups $(15.2 \mathrm{vs}$ $10.3 \%, P=0.07 ; 23.5$ vs $13.1 \%, P<0.01 ; 13.8$ vs $9.7 \%$,
$P=0.08$; for the presence of early ARM, RPE abnormalities, and soft indistinct or reticular drusen, respectively). 
Table 3 shows a comparison of the person-specific late and early ARM prevalence between this surgical cohort and the BMES population by age group. The prevalence of late ARM in the BMES population was nonsignificantly higher than in this surgical cohort (2.8 vs $1.9 \%, P=0.37)$. The proportions with early ARM or early ARM component lesions (RPE abnormalities and soft indistinct or reticular drusen) were significantly higher in this surgical cohort than in the older subgroup of the BMES population (14.5 vs 6.9\%, $P<0.01 ; 23.6$ vs $13.2 \%$, $P<0.01$; and 12.8 vs $5.8 \%, P<0.01$; for the presence of early ARM, RPE abnormalities, and soft indistinct or reticular drusen, respectively).

Table 4 presents a comparison of the person-specific ARM prevalence of this surgical cohort with the BMES older subgroups after age standardization to the BMES population. This demonstrated a significantly lower frequency of late ARM (0.9 vs 2.8\%, $P$-value $<0.05)$, but a significantly higher frequency of early ARM (9.7 vs 6.9\%,
$P$-value $<0.05)$ in this surgical cohort compared to the older subgroup of the BMES population.

\section{Discussion}

In a hospital-based sample of cataract surgery patients, we found that early ARM and early ARM component lesions (RPE abnormalities and soft indistinct or reticular drusen), but not late ARM, were more common in surgical than in nonsurgical eyes. We also found that the early ARM prevalence in our surgical cohort was higher than that in the older subgroup of the BMES population with a similar age range. The lower prevalence of late ARM in the surgical cohort compared with the nonsurgical eyes or the BMES population could have been the result of surgical selection: patients with late ARM are less likely to be recommended for cataract surgery.

Our findings are in agreement with a previous prospective population-based report that showed an

Table 3 Comparison of prevalence of ARM between this surgical cohort and the older subgroup of the BMES population

\begin{tabular}{|c|c|c|c|c|c|c|c|c|c|}
\hline \multirow[t]{3}{*}{ Characteristics, eye } & \multicolumn{8}{|c|}{ Age group (years) } & \multirow[b]{3}{*}{ P-value } \\
\hline & \multicolumn{2}{|c|}{$60-69$} & \multicolumn{2}{|c|}{$70-79$} & \multicolumn{2}{|c|}{$80+$} & \multicolumn{2}{|c|}{ Total } & \\
\hline & $\mathrm{n}$ & $\%$ & $\mathrm{n}$ & $\%$ & $\mathrm{n}$ & $\%$ & $\mathrm{n}$ & $\%$ & \\
\hline \multicolumn{10}{|l|}{ Late ARM } \\
\hline Surgical eye $(n=412)$ & 0 & 0 & 1 & 0.5 & 7 & 5.2 & 8 & 1.9 & 0.37 \\
\hline BMES $(n=2579)$ & 6 & 0.5 & 25 & 2.7 & 41 & 12.0 & 72 & 2.8 & \\
\hline \multicolumn{10}{|l|}{ Early ARM } \\
\hline Surgical eye $(n=399)$ & 5 & 5.6 & 19 & 10.2 & 34 & 27.6 & 58 & 14.5 & $<0.01$ \\
\hline BMES $(n=2411)$ & 47 & 3.7 & 82 & 9.4 & 38 & 14.1 & 167 & 6.9 & \\
\hline \multicolumn{10}{|l|}{ RPE abnormalities } \\
\hline Surgical eye $(n=399)$ & 12 & 13.5 & 37 & 19.8 & 45 & 36.6 & 94 & 23.6 & $<0.01$ \\
\hline BMES $(n=2487)$ & 116 & 9.1 & 141 & 15.6 & 72 & 24.2 & 329 & 13.2 & \\
\hline \multicolumn{10}{|c|}{ Indistinct soft drusen/reticular drusen } \\
\hline Surgical eye $(n=399)$ & 4 & 4.5 & 15 & 8.0 & 32 & 26.0 & 51 & 12.8 & $<0.01$ \\
\hline $\operatorname{BMES}(n=2411)$ & 37 & 2.9 & 70 & 8.0 & 33 & 12.3 & 140 & 5.8 & \\
\hline
\end{tabular}

$\mathrm{RPE}=$ retinal pigment epithelial.

Table 4 Comparison of ARM prevalence between this surgical cohort and the older subgroup of the BMES population, after direct age standardization to the BMES population

$$
\% \text { of Participants }
$$

\begin{tabular}{lccc}
\cline { 2 - 4 } Characteristics & Study population & BMES population & Age-adjusted P-value \\
\hline Late ARM & 0.9 & 2.8 & $<0.05$ \\
Early ARM & 9.7 & 6.9 & $<0.05$ \\
RPE abnormalities & 18.5 & 13.2 & $<0.01$ \\
Indistinct soft drusen/reticular drusen & 8.2 & 5.8 & 0.054 \\
\hline
\end{tabular}

$\mathrm{RPE}=$ retinal pigment epithelial. 
increase in the incidence of early ARM in the shortmedium term $\left(<5\right.$ years) after cataract surgery. ${ }^{14}$ Our findings may also complement previous findings from large population-based cross-sectional and longitudinal studies showing an association between past cataract surgery and subsequent development of late ARM in a long term. ${ }^{13-15,22}$ It is plausible that patients who undergo cataract surgery have an increased risk of developing early ARM in the short-medium term (1-3 years), which then progresses to late ARM over a longer term (at least 5 years). Further follow-up of this cohort 5-6 years after surgery would be useful to confirm or deny this possibility.

An alternative explanation for our findings should be considered. Selection bias could have played a role, as patients who undergo cataract surgery may have visual impairment not purely due to cataract but also due to coexisting ocular pathologies, including early ARM. These patients could have been more likely to be recommended for cataract surgery as a result of their impaired vision. The finding of a higher early ARM frequency in surgical than in nonsurgical eyes would then result from such surgical selection, although the typical bilateral nature of ARM could argue against this explanation. ${ }^{23}$ In support, our previous report of baseline characteristics of this surgical cohort, ${ }^{24}$ collected from medical records, showed that the preoperative prevalence of any ARM in this sample was similar to BMES participants with and without a history of previous cataract surgery $(8.7 \%$ in this study sample $v \mathrm{~s}$ 9.0 and $8.8 \%$ in the BMES population with and without history of previous surgery, respectively). We further analysed the proportions with preoperative late ARM (presence of neovascular ARM or geographic atrophy) and early ARM (presence of soft drusen or RPE abnormalities) in this sample of eligible patients $(n=671)$ and found a similar rate to the BMES population, after age standardisation (1.6 vs $2.8 \%$ for late ARM, and 8.3 vs $6.9 \%$ for early ARM; for this study sample vs BMES population, respectively) (data not shown).

A second alternative explanation is that our findings could suggest that cataract and early ARM are associated. It has been postulated that cataract and ARM might occur frequently together because of shared genetic ${ }^{25,26}$ or environmental factors, cohort including age, diet, ${ }^{27,28}$ light exposure ${ }^{29,30}$ and cigarette smoking. ${ }^{31-36}$ Current available evidence, however, is weak and inconsistent ${ }^{11,12,14,15}$ in support of an association between cataract and ARM.

If future studies provide additional evidence supporting an association between prior cataract surgery and the subsequent development of early and late ARM, research will be needed to elucidate mechanisms behind this association. Possible mechanisms include retinal inflammatory changes associated with surgery, ${ }^{10,37}$ increased light exposure after removal of the lens, ${ }^{7}$ and a postoperative biochemical environment in operated eyes (increased free radicals or growth factors), $7,10,38$ which could accelerate ARM progression.

Overall, we achieved a reasonable follow-up rate: $64 \%$ of all eligible baseline participants (454/709) or $73 \%$ of surviving eligible participants with available preoperative data (454/622). The preoperative characteristics of those who were followed and those who refused to participate were similar, indicating that persons lost to follow-up would have been unlikely to substantially alter the study findings. Standardized retinal photographs were taken at follow-up examinations and masked grading of ARM lesions was performed by a grader who achieved good inter- and intragrader reliability. ${ }^{19}$ Weaknesses in this study include a lack of baseline retinal photographic documentation of the macula and potential selection biases inherent with cataract surgical patients.

In summary, we found that eyes that had undergone cataract surgery were more likely in the short-medium term to have early ARM compared to paired eyes that did not undergo surgery, and to the BMES population with a similar age range. These findings are in agreement with reported data from the 5-year follow-up of the BDES, ${ }^{14}$ and may complement earlier findings of an increased long-term incidence of late ARM after cataract surgery. ${ }^{13}$ Further long-term investigations are needed to clarify whether the observed increase in early ARM prevalence in the short-medium term after surgery leads to an increase in later ARM prevalence in the longer term.

\section{References}

1 Rahmani B, Tielsch JM, Katz J, Gottsch J, Quigley H, Javitt J et al. The cause-specific prevalence of visual impairment in an urban population. The Baltimore Eye Survey. Ophthalmology 1996; 103(11): 1721-1726.

2 Klaver CC, Wolfs RC, Vingerling JR, Hofman A, de Jong PT. Age-specific prevalence and causes of blindness and visual impairment in an older population: the Rotterdam Study. Arch Ophthalmol 1998; 116(5): 653-658.

3 Javitt JC, Wang F, West SK. Blindness due to cataract: epidemiology and prevention. Annu Rev Public Health 1996; 17: 159-177.

4 Attebo K, Mitchell P, Smith W. Visual acuity and the causes of visual loss in Australia. The Blue Mountains Eye Study. Ophthalmology 1996; 103(3): 357-364.

5 Klein R, Wang Q, Klein BE, Moss SE, Meuer SM. The relationship of age-related maculopathy, cataract, and glaucoma to visual acuity. Invest Ophthalmol Vis Sci 1995; 36(1): 182-191. 
6 Blair CJ, Ferguson Jr J. Exacerbation of senile macular degeneration following cataract extraction. Am J Ophthalmol 1979; 87(1): 77-83.

7 Pollack A, Marcovich A, Bukelman A, Oliver M. Agerelated macular degeneration after extracapsular cataract extraction with intraocular lens implantation. Ophthalmology 1996; 103(10): 1546-1554.

8 Pollack A, Marcovich A, Bukelman A, Zalish M, Oliver M. Development of exudative age-related macular degeneration after cataract surgery. Eye 1997; 11(Part 4): 523-530.

9 Chaine G, Hullo A, Sahel J, Soubrane G, Espinasse-Berrod MA, Schutz D et al. Case-control study of the risk factors for age related macular degeneration. France-DMLA study group. Br J Ophthalmol 1998; 82(9): 996-1002.

10 van der Schaft TL, Mooy CM, de Bruijn WC, Mulder PG, Pameyer JH, de Jong PT. Increased prevalence of disciform macular degeneration after cataract extraction with implantation of an intraocular lens. Br J Ophthalmol 1994; 78(6): 441-445.

11 Wang JJ, Mitchell PG, Cumming RG, Lim R. Cataract and age-related maculopathy: the Blue Mountains Eye Study. Ophthalmic Epidemiol 1999; 6(4): 317-326.

12 Klein R, Klein BE, Wang Q, Moss SE. Is age-related maculopathy associated with cataracts? Arch Ophthalmol 1994; 112(2): 191-196.

13 Wang JJ, Klein R, Smith W, Klein BE, Tomany S, Mitchell P. Cataract surgery and the 5-year incidence of late-stage agerelated maculopathy: pooled findings from the Beaver Dam and Blue Mountains Eye Studies. Ophthalmology 2003; 110(10): 1960-1967.

14 Klein R, Klein BE, Jensen SC, Cruickshanks KJ. The relationship of ocular factors to the incidence and progression of age-related maculopathy. Arch Ophthalmol 1998; 116(4): 506-513.

15 Klein R, Klein BE, Wong TY, Tomany SC, Cruickshanks KJ. The association of cataract and cataract surgery with the long-term incidence of age-related maculopathy: the Beaver Dam Eye Study. Arch Ophthalmol 2002; 120(11): 1551-1558.

16 Braunstein RE, Sparrow JR. A blue-blocking intraocular lens should be used in cataract surgery. Arch Ophthalmol 2005; 123(4): 547-549.

17 Mainster MA. Intraocular lenses should block UV radiation and violet but not blue light. Arch Ophthalmol 2005; 123(4): 550-555.

18 ETDRS Coordinating Center UoMDoEaPM. Early Treatment Diabetic Retinopathy Study (ETDRS). Manual of Operation. Springfield, VA 22161, National Technical Information Service: (accession number \#PB85223006), 1980.

19 Mitchell P, Smith W, Attebo K, Wang JJ. Prevalence of agerelated maculopathy in Australia. The Blue Mountains Eye Study. Ophthalmology 1995; 102(10): 1450-1460.

20 Klein R, Davis M, Magli Y, Segal P, Klein B, Hubbard L. The Wisconsin age-related maculopathy grading system. Ophthalmology 1991; 98: 1128-1134.

21 Mitchell P, Cumming RG, Attebo K, Panchapakesan J. Prevalence of cataract in Australia: the Blue Mountains Eye Study. Ophthalmology 1997; 104(4): 581-588.
22 Freeman EE, Munoz B, West SK, Tielsch JM, Schein OD. Is there an association between cataract surgery and agerelated macular degeneration? Data from three populationbased studies. Am J Ophthalmol 2003; 135(6): 849-856.

23 Wang JJ, Mitchell P, Smith W, Cumming RG. Bilateral involvement by age related maculopathy lesions in a population. Br J Ophthalmol 1998; 82(7): 743-747.

24 Pham TQ, Wang JJ, Rochtchina E, Maloof A, Mitchell P. Systemic and ocular comorbidity of cataract surgical patients in a western Sydney public hospital. Clin Exp Ophthalmol 2004; 32(4): 383-387.

25 Heiba IM, Elston RC, Klein BE, Klein R. Genetic etiology of nuclear cataract: evidence for a major gene. Am J Med Genet 1993; 47(8): 1208-1214.

26 Heiba IM, Elston RC, Klein BE, Klein R. Sibling correlations and segregation analysis of age-related maculopathy: the Beaver Dam Eye Study. Genet Epidemiol 1994; 11(1): 51-67.

27 Mares-Perlman JA, Klein R, Klein BE, Greger JL, Brady WE, Palta $\mathrm{M}$ et al. Association of zinc and antioxidant nutrients with age-related maculopathy. Arch Ophthalmol 1996; 114(8): 991-997.

28 Mares-Perlman JA, Lyle BJ, Klein R, Fisher AI, Brady WE, VandenLangenberg GM et al. Vitamin supplement use and incident cataracts in a population-based study. Arch Ophthalmol 2000; 118(11): 1556-1563.

29 Taylor HR, West S, Munoz B, Rosenthal FS, Bressler SB, Bressler NM. The long-term effects of visible light on the eye. Arch Ophthalmol 1992; 110: 99-104.

30 Cruickshanks KJ, Klein BE, Klein R. Ultraviolet light exposure and lens opacities: the Beaver Dam Eye Study. Am J Public Health 1992; 82(12): 1658-1662.

31 Cumming RG, Mitchell P. Alcohol, smoking, and cataracts: the Blue Mountains Eye Study. Arch Ophthalmol 1997; 115(10): 1296-1303.

32 Klein BE, Klein R, Linton KL, Franke T. Cigarette smoking and lens opacities: the Beaver Dam Eye Study. Am J Prev Med 1993; 9(1): 27-30.

33 Christen WG, Manson JE, Seddon JM, Glynn RJ, Buring JE, Rosner B et al. A prospective study of cigarette smoking and risk of cataract in men. JAMA 1992; 268(8): 989-993.

34 Smith W, Mitchell P, Leeder SR. Smoking and age-related maculopathy. The Blue Mountains Eye study. Arch Ophthalmol 1996; 114(12): 1518-1523.

35 Vingerling JR, Hofman A, Grobbee DE, de Jong PT. Agerelated macular degeneration and smoking. The Rotterdam Study. Arch Ophthalmol 1996; 114(10): 1193-1196.

36 Klein R, Klein BE, Moss SE. Relation of smoking to the incidence of age-related maculopathy. The Beaver Dam Eye Study. Am J Epidemiol 1998; 147(2): 103-110.

37 Anderson DH, Mullins RF, Hageman GS, Johnson LV. A role for local inflammation in the formation of drusen in the aging eye. Am J Ophthalmol 2002; 134(3): 411-431.

38 Penfold PL, Provis JM, Billson FA. Age-related macular degeneration: ultrastructural studies of the relationship of leucocytes to angiogenesis. Graefes Arch Clin Exp Ophthalmol 1987; 225(1): 70-76. 